\title{
The changing clinical spectrum of Malaria: a clinical study from Bundelkhand
}

\author{
Singh Neeshu\#, Mathur Aman ${ }^{\#}$,Naim Faran ${ }^{*}$,Agarwal Nutan** \\ \# senior resident,department of Medicine,M.L.B Medical College,Jhansi \\ * senior resident,department of Medicine,Mujjafarnagar Medical College,Mujjafarnagar \\ **professor,department of medicine,MLB Medical College,Jhansi
}

\begin{abstract}
Objective: To find out clinical and laboratory findings in acute malaria caused by various plasmodium species.

Methods: This was a prospective study which was carried out in the department of medicine at MLB Medical College, Jhansi from May 2012 to September 2013.In this prospective study,401 patients of malaria were enrolled who met both inclusion and exclusion criteria. Frequencies of alterations in clinical and laboratory parameters were determined in various plasmodium species.

Results: Out of 401 patients, 194 were Plasmodium falciparum, 185 were P.vivax and 22 were mixed infection. Fever (97.5\%), chills and rigors (84.7\%) were the most common complaint in all three varieties of plasmodium infection. Pallor (78.3\%) and splenomegaly (56.6\%) were often detected in individuals with malaria. Thrombocytopenia (52.8\%) was most common laboratory abnormalities detected in various plasmodium species. Jaundice and anemia were more common in P.falciparum and mixed infection as compared to P.vivax

Conclusion: Malaria must be considered as leading differential diagnosis in an acutely febrile patient with one or more of abnormalities like splenomegaly, fall in blood counts or rise in bilirubin etc. Fever with chills and rigors, pallor, splenomegaly and thrombocytopenia were most common manifestation in malaria.
\end{abstract}

Keywords: Malaria, clinical spectrum.

\section{Introduction}

Malaria is the most important parasitic disease. Species of plasmodium that cause this deadly female anopheles transmitted disease are P. vivax, P. falciparum, P. ovale, P. malariae and P. knowlesi. Based on documented cases, the WHO estimates that there were 216 million cases of malaria in 2010 resulting in 655,000 deaths. This is equivalent to roughly 2000 deaths every day. A 2012 study estimated the number of documented and undocumented deaths in 2010 was 1.24 million.

Malaria is not a uniform disease; it encompasses many manifestations and its impact varies on epidemiological setting. It usually presents as fever, chills with rigor malaise, headache, myalgia, anorexia vomiting, jaundice, anaemia. Clinically, it may present with hepatosplenomegaly, acute renal failure, gastrointestinal symptoms, dehydration, shock, cerebral malaria and black water fever.

\section{Material And Methods}

This was a prospective study which was carried out in department of medicine at MLB Medical college, Jhansi from may 2012 to September 2013.In this prospective study ,401 patients of malaria were enrolled who met both inclusion and exclusion criteria. . Out of 401 patients ,194 were Plasmodium falciparum, 185 were P.vivax and 22 were mixed infection .After establishing the diagnosis ,clinical evaluation was done and information regarding fever, chills, rigors, vomiting, headache, diarrhoea, altered sensorium and various laboratory parameters were recorded.

\section{Results}

Table 1 shows various clinical features of different plasmodium species. Fever was present in $97.5 \%$ of the total patients. Fever was the presenting complaint in $98 \%$ of patients with P.falciparum malaria and $97 \%$ of patients with P.vivax malaria. It was present in $90 \%$ of cases of mixed malarial infection. It was most common manifestation of malaria.

Chills and rigors were present in $84.7 \%$ of total patients. It was a presenting complaint in $89.6 \%$ of patients with P.falciparum malaria. It was presents in $81.8 \%$ cases of mixed malarial infection. It was also seen in $80 \%$ of patients with P.vivax malaria.

Headache was presenting complaints in $55.6 \%$ of the patients. It was more common in P.falciparum (63.4\%). It was also present $49.1 \%$ in P.vivax malaria and $40 \%$ of mixed malarial infection.

Altered Sensorium: Altered sensorium was seen in $46.6 \%$ of the patients. It was not noted in any of the patients with vivax malaria. It was seen in $51.5 \%$ of the patients with falciparum infection, in about 
$46.4 \%$ of the patients with vivax malaria and $45 \%$ with mixed infection.

Nausea and Vomiting: Nausea and vomiting was one of the frequent symptoms in malarial infection. It was seen in $27.1 \%$ of the total cases. Whereas the symptoms was observed in $32.4 \%$ and $22.7 \%$ of patients with falciparum and vivax respectively.

Diarrhoea: it was observed in $4.9 \%$ of the total cases. It was more common in P.falciparum malaria (6.1\%).

Table 2 shows various clinical signs of malaria in different plasmodium species. Pallor was more common in mixed infection (86.3\%).It was seen in $81.5 \%$ and $64.3 \%$ patients of Falciparum and Vivax malaria respectively. It was the most common clinical sign of malaria.

Splenomegaly was the second most important clinical sign in our study.

It was more common in Falciparum malaria $(58.7 \%)$.There was no significant difference between vivax and mixed infection.

Icterus was equally present in both Falciparum and Vivax malaria (32.9\%).It was present in $9 \%$ of cases of mixed infection.

Hypotension was more common with mixed infection (50\%).It was seen in $43.2 \%$ and $18.9 \%$ patients of Falciparum and Vivax malaria respectively.

Cerebral malaria was more common with falciparum malaria (45.3\%). It was $15.1 \%$ and $9 \%$ in Vivax and mixed infection malaria respectively.

Hypoglycaemia was more common with mixed infection (36.5\%).It was seen in $29.8 \%$ and $2.1 \%$ patients of Falciparum and Vivax malaria respectively.

Hepatomegaly was more common with falciparum malaria (12.3\%). It was seen in $9 \%$ and $4.3 \%$ patients of mixed infection and vivax malaria respectively.

\section{Laboratory parameters:}

Table 3 shows various haematological abnormalities in different plasmodium species. Out of 401 total malaria cases, severe anaemia (i.e. haemoglobin $<7 \mathrm{mg} \%$ ) was seen in $99(24.6 \%)$ patients. Among patients with severe anaemia 57(29.3\%) were P. falciparum cases, 38(20.5\%) were P. vivax cases, and 4(18.1\%) were having mixed infection. Severe anaemia was more common with falciparum malaria.

Out of 401 total patients, 212 patients (i.e.52.8\%) had platelet count $<50000 / \mu l$ out of which 110(56.7\%) were of Falciparum, 95(51.3\%) were Vivax malaria and 7(31.8\%) of mixed infection. Thrombocytopenia was more common with falciparum malaria.

Out of 401 total patients, 105 patients (i.e. $26.1 \%$ ) had leukocyte count $<4000 / \mu l, 224$ patients (i.e. $55.86 \%$ ) had counts leukocyte between $4000-11000 / \mu l$ and, 72 patients (i.e. 17.9\%) had leukocyte count $>11,000 / \mu \mathrm{l}$.

Leucopoenia was more common with falciparum malaria.Leukocytosis was equally common in different types of malaria.

\section{Biochemical Profile}

Table 4 shows various laboratory abnormalities in different plasmodium species. Out of 401 total malaria cases severe jaundice (i.e. bilirubin $>10 \mathrm{mg} \%$ ) was seen in $28(6.9 \%)$ of patients. Among patients with severe jaundice $14(7.2 \%)$ were P. falciparum cases, $12(6.4 \%)$ were P. vivax cases and 2(9\%) were having mixed infection. Severe jaundice was more common with mixed infection.

Out of total 401 malaria patients SGOT levels were done in 364 patients. Out of 364 patients, 154 patients (i.e. 42.3\%) had SGOT levels between 40-100 IU/L, and 122 patients (i.e. 33.5\%) had SGOT levels $>100 \mathrm{IU} / \mathrm{L}$.

It was more deranged in falciparum malaria (33.5\%) It was seen in $31.8 \%$ and $27 \%$ patients of mixed infection and vivax malaria respectively.

Out of total 401 malaria patients, 169 patients (i.e. $42.1 \%$ ) had SGPT levels between 40-100 IU/L, and 129 patients (i.e. $32.1 \%$ ) had SGPT levels $>100$ IU/L. It was more deranged with falciparum malaria $(36 \%)$

Deranged level was seen in $31.8 \%$ and $28 \%$ of patients mixed infection and vivax malaria respectively.

Out of total 401patients, 244 patients (60.8\%) had serum creatinine <1.4, 104 patients $(25.9 \%)$ had serum creatinine value of 1.4 to 2.5 , and 53 patients $(13.2 \%)$ had serum creatinine value of $>2.6$. Among patients with severe renal failure $34(17.5 \%)$ were P. falciparum cases, $17(9.1 \%)$ were P. vivax cases and $2(9 \%)$ were having mixed infection. Severe renal failure was more common with falciparum malaria.

\section{Discussion}

In present prospective study, 401 cases of malaria were taken who met both inclusion and exclusion criteria.

- Out of 401 cases $48.37 \% \%$ were of P. Falciparum, $46.1 \%$ were of P.vivax and 5.4\% were of mixed infection 
- $\quad$ Fever $(97.5 \%)$, chills and rigors $(84.7 \%)$ were most common complaint, other common symptoms were headache $(55.6 \%)$ and altered sensorium $(48.8 \%)$.infrequent complaints were nausea or vomiting $(27.1 \%)$ and diarrhoea $(4.9 \%)$.

- Most common clinical sign was pallor (783\%) and splenomegaly (56.6\%), other common clinical signs were icterus (31.6\%), hypotension (32.4\%), cerebral malaria (29.4\%) and hypoglycaemia (17.4\%).infrequent clinical sign was hepatomegaly $(8.4 \%)$

- On laboratory investigations, severe anaemia and thrombocytopenia were most common abnormalities detected. Hyperbilirubinemia $(>10 \mathrm{mg} \%)$ was detected in $6.9 \%$ of patients. Severe renal failure (s.creatinine $>2.6 \mathrm{mg} \%$ ) was $13.2 \%$ of cases.

Conclusion: Clinical spectrum of malaria of different species was studied. Fever with chills and rigors, pallor, splenomegaly, anaemia and thrombocytopenia were most common.

Table-1: Incidence of various symptoms

\begin{tabular}{|c||c||c||c||c||}
\hline \hline Symptoms & $\begin{array}{c}\text { Pl.falciparum } \\
(\mathbf{n = 1 9 4 )}\end{array}$ & $\begin{array}{c}\text { Pl. vivax } \\
(\mathbf{n}=\mathbf{1 8 5})\end{array}$ & $\begin{array}{c}\text { Mixed infection } \\
(\mathbf{n}=\mathbf{2 2})\end{array}$ & $\begin{array}{c}\text { TOTAL } \\
(\mathbf{n}=\mathbf{4 0 1})\end{array}$ \\
\hline \hline Fever & $191(98.4 \%)$ & $180(97.2 \%)$ & $20(90 \%)$ & $\mathbf{3 9 1}(\mathbf{9 7 . 5 \%})$ \\
\hline \hline Chills and rigors & $174(89.6 \%)$ & $148(80 \%)$ & $18(81.8 \%)$ & $\mathbf{3 4 0}(\mathbf{8 4 . 7 \%})$ \\
\hline \hline Headache & $123(63.4 \%)$ & $91(49.1 \%)$ & $9(40 \%)$ & $\mathbf{2 2 3}(\mathbf{5 5 . 6 \%})$ \\
\hline \hline Altered sensorium & $100(51.5 \%)$ & $86(46.4 \%)$ & $10(45 \%)$ & $\mathbf{1 9 6}(\mathbf{4 8 . 8 \%})$ \\
\hline \hline Nausea and vomiting & $63(32.4 \%)$ & $42(22.7 \%)$ & $4(18.1 \%)$ & $\mathbf{1 0 9}(\mathbf{2 7 . 1 \%})$ \\
\hline \hline Diarrhoea & $12(6.1 \%)$ & $8(4.3 \%)$ & $0(0 \%)$ & $\mathbf{2 0}(\mathbf{4 . 9 \%})$ \\
\hline
\end{tabular}

Table: 2 Incidence of various clinical signs.

\begin{tabular}{|c|c|c|c|l|}
\hline Signs & $\begin{array}{c}\text { P.falciparum } \\
(\mathbf{n = 1 9 4 )}\end{array}$ & $\begin{array}{c}\text { P.vivax } \\
(\mathbf{n = 1 8 5})\end{array}$ & $\begin{array}{c}\text { Mixed infection } \\
(\mathbf{n = 2 2})\end{array}$ & $\begin{array}{l}\text { Total } \\
(\mathbf{n}-\mathbf{4 0 1})\end{array}$ \\
\hline Pallor & $158(81.5 \%)$ & $119(64.3 \%)$ & $19(86.3 \%)$ & $296(73.8 \%)$ \\
\hline Splenomegaly & $114(58.7 \%)$ & $101(54.5 \%)$ & $12(54.0 \%)$ & $227(56.6 \%)$ \\
\hline Icterus & $64(32.9 \%)$ & $61(32.9 .3 \%)$ & $2(9 \%)$ & $127(31.6 \%)$ \\
\hline Hypotension & $84(43.2 \%)$ & $35(18.9 \%)$ & $11(50 \%)$ & $130(32.4 \%)$ \\
\hline Cerebral malaria & $88(45.3 \%)$ & $28(15.1 \%)$ & $2(9 \%)$ & $118(29.4 \%)$ \\
\hline Hypoglycaemia & $58(29.8 \%)$ & $4(2.1 \%)$ & $8(36.3 \%)$ & $70(17.4 \%)$ \\
\hline Hepatomegaly & $24(12.3 \%)$ & $8(4.3 \%)$ & $2(9 \%)$ & $34(8.4 \%)$ \\
\hline
\end{tabular}

Table: 3 Hematological Profiles

\begin{tabular}{|l|l|l|l|l|}
\hline PARAMETERS & P.FALCIPARUM & P.VIVAX & MIXED INFECTION & TOTAL \\
\hline HEMOGLOBIN & \multicolumn{5}{|l|}{} \\
\hline$>10 \mathrm{mg} \%$ & $60(30.9 \%)$ & $87(47 \%)$ & $10(45 \%)$ & $157(39.1 \%)$ \\
\hline $7-10 \mathrm{mg} \%$ & $78(40.2 \%)$ & $60(32.4 \%)$ & $8(36 \%)$ & $146(36.4 \%)$ \\
\hline$<7 \mathrm{mg} \%$ & $38(20.5 \%)$ & $4(18.1 \%)$ & $99(24.6 \%)$ \\
\hline PLATELET COUNT & $57(29.3 \%)$ & $42(22.7 \%)$ & $5(22 \%)$ & $77(19.2 \%)$ \\
\hline$>11$ lakh & $30(15.4 \%)$ & $48(25.9 \%)$ & $10(45.4 \%)$ & $112(27.9 \%)$ \\
\hline $50,000-1$ lakh & $54(27 \%)$ & $95(51.3 \%)$ & $7(31.8 \%)$ & $212(52.8 \%)$ \\
\hline$<50,000$ & $110(56.7 \%)$ & $39(21 \%)$ & $6(27.2 \%)$ & $105(26.1 \%)$ \\
\hline LEUKOCYTE COUNT & $60(31 \%)$ & $114(61.6 \%)$ & $12(54.5 \%)$ & $224(55.8 \%)$ \\
\hline$<4,000$ & $98(50.5 \%)$ & $32(17.2 \%)$ & $4(18.1 \%)$ & $72(17.9 \%)$ \\
\hline $4,000-11,000$ & $36(18.5 \%)$ &
\end{tabular}

Table: 4 Biochemical Profile

\begin{tabular}{|l|l|l|l|l|}
\hline PARAMETERS & P.FALCIPARUM & P.VIVAX & MIXED INFECTION & TOTAL \\
\hline SERUM BILIRUBIN & $123(63.4 \%)$ & $135(72.9 \%)$ & $16(72.7)$ & $274(68.3 \%)$ \\
\hline $1-3 \mathrm{mg} \%$ & $57(29.3 \%)$ & $38(20.5 \%)$ & $4(18.1 \%)$ & $99(24.6 \%)$ \\
\hline $3-10 \mathrm{mg} \%$ & $14(7.2 \%)$ & $12(6.4 \%)$ & $2(9 \%)$ & $28(6.9 \%)$ \\
\hline$>10 \mathrm{mg} \%$ & $78(40 \%)$ & $69(37.2 \%)$ & $7(31.8 \%)$ & $154(38.4 \%)$ \\
\hline SGOT & $65(33.5 \%)$ & $50(27 \%)$ & $7(31.8 \%)$ & $122(30.4 \%)$ \\
\hline $40-100$ & $86(44.3 \%)$ & $74(40 \%)$ & $9(40 \%)$ & $169(42.1 \%)$ \\
\hline$>100$ & $70(36 \%)$ & $52(28 \%)$ & $7(31.8 \%)$ & $129(32.1 \%)$ \\
\hline SGPT & \multicolumn{5}{|l|}{} \\
\hline $40-100$ & $98(50.5 \%)$ & $131(70.8 \%)$ & $15(68 \%)$ & $244(60.8 \%)$ \\
\hline$>100$ & $62(31.9 \%)$ & $37(20 \%)$ & $5(22.7 \%)$ & $104(25.9 \%)$ \\
\hline SERUM CREATININE & $17(9.1 \%)$ & $2(9 \%)$ & $53(13.2 \%)$ \\
\hline$<1.4$ & $34(17.5 \%)$ &
\end{tabular}




\section{References}

[1]. Harrison Principles of Internal Medicine, $18^{\text {th }}$ Ed.; Nicholas J. White, Joel G Bremani: 1688-1694

[2]. Bashawri LAM, Mandil AA, Bahnassy AA, Ahmed MA. Malaria: Haematological Aspects. Annals of Saudi Medicine 2002; $22: 372-7$

[3]. Biswas R, Sengupta G, Mundle M. A Controlled Study on Haemograms of Malaria Patients in Calcutta. Indian J Malariol.1999;36:42-8

[4]. Kochar DK et al; Cerebral Malaria in Indian adults a prospective study; JAPI 2002; 50; 234-41.

[5]. Jadhav UM, Patkar VS, Kadam NN. Thrombocytopenia in Malaria- Correlation with Type and Severity of Malaria. J Assoc Physicians India.2004;52:615-8

[6]. Mehta: Clinical pattern of Malaria epidemics in Rajasthan; Journal of Physicians of India 2001; 48; 211-215.

[7]. Murthy: Malarial hepatitis - Does such a Clinical entity exist : Journal of Am and association of physician of India vol.47 : No.1:27.

[8]. Kochar et al., Cerebral malaria in Indian adults; a prospective study of 441 patients from Bikaner. J Assoc Physicians India, 2002. 50: 234-41 